\title{
OBSTRUCTIONS TO DEFORMING A SPACE CURVE
}

\author{
BY
}

DANIEL J. CURTIN

\begin{abstract}
Mumford described a curve, $\gamma$, in $\mathbf{P}^{3}$ that has obstructed infinitesimal deformations (in fact the Hilbert scheme of the curve is generically nonreduced). This paper studies $\gamma$ 's Hilbert scheme by studying deformations of $\gamma$ in $\mathbf{P}^{3}$ over parameter spaces of the form $\operatorname{Spec}\left(k[t] /\left(t^{n}\right)\right), n=2,3, \ldots$ Given a deformation of $\gamma$ over $\operatorname{Spec}\left(k[t] /\left(t^{n}\right)\right)$ one attempts to extend it to a deformation of $\gamma$ over $\operatorname{Spec}\left(k[t] /\left(t^{n+1}\right)\right)$. If it will not extend, this deformation is said to be obstructed at the nth order.

I show that on a generic version of Mumford's curve, an infinitesimal deformation (i.e., a deformation over $\operatorname{Spec}\left(k[t] /\left(t^{2}\right)\right)$ ) is either obstructed at the second order, or at no order, in which case we say it is unobstructed.
\end{abstract}

Let $F$ be a nonsingular cubic surface in $\mathrm{P}^{3}$, let $E$ be one of the 27 lines on $F$, and let $H$ be the hyperplane divisor on $F$. Mumford [3] showed that any nonsingular member $\gamma$ of the linear system $|4 H+2 E|$ represents a point of the Hilbert scheme $\mathbf{H}$ parametrizing curves of degree 14 and genus 24 at which $\mathbf{H}$ is not reduced. This can be restated in the language of infinitesimal deformations. An infinitesimal deformation of $\gamma$ is a flat family of projective space curves over $\operatorname{Spec}\left(k[t] /\left(t^{2}\right)\right)$ with $\gamma$ as special fiber. One attempts to lift such a deformation successively to deformations over $\operatorname{Spec}\left(k[t] /\left(t^{n}\right)\right), n=3,4, \ldots$ If there is an $n$ such that the deformation extends modulo $t^{n}$, but not modulo $t^{n+1}$, we say the deformation is obstructed at the nth order. The fact that the Hilbert scheme is nonreduced can be interpreted as saying that $\gamma$ has infinitesimal deformations that are obstructed at some order. More specifically, Mumford showed that the dimension of $\mathbf{H}$ at the point $z_{\gamma}$ representing $\gamma$ is 56 while the dimension of the tangent space to $\mathbf{H}$ at $z_{\gamma}$ is 57. This guarantees the existence of infinitesimal deformations that are obstructed.

I will show that for a nonsingular element $\gamma$ of $|4 H+2 E|$ any infinitesimal deformation either is obstructed at the second order, or at no order (in which case the deformation is said to be unobstructed).

In $\S 1$, the generators and relations of the ideal defining a member of $|4 H+2 E|$ are given. From these come a free resolution of the ideal, and a resolution of the structure sheaf of the nonsingular members. In $\$ 2$, the deformations of $\gamma$ in $\mathbf{P}^{3}$ are related to the homogeneous deformations of certain affine cones over $\gamma$. In $\$ 3$, the unobstructed deformations of $\gamma$ are obtained from the $2 \times 2$ minors of a $2 \times 4$ matrix of polynomials. In $\S 4$ and $\$ 5$, the obstructed deformations are identified

Received by the editors April 28, 1980 and, in revised form, September 12, 1980.

1980 Mathematics Subject Classification. Primary 14H10, 14C05, 14 D15.

Key words and phrases. Projective space curves, infinitesimal deformations, obstructed deformations, deformations of cones, Hilbert scheme.

(c) 1981 American Mathematical Society $0002-9947 / 81 / 0000-0405 / \$ 04.00$ 
and the cohomology classes representing the second order obstructions are found. Finally (Theorem 2), these classes are seen to actually obstruct the deformations at the second order, at least on a general $\gamma$.

I thank Michael Schlessinger, my thesis advisor, for his help and encouragement.

Conventions. $k$ is an algebraically closed field, $P=k\left[x_{0}, \ldots, x_{n}\right], \underline{x}=$ $x_{0}, \ldots, x_{n}$ (usually $n=3$ and we use $x_{0}=x, x_{1}=y, x_{2}=z, x_{3}=w$ ), $P_{s}=$ forms of degree $s$ in $P$. $P(i)$ is defined by $P(i)_{s}=P_{i+s}$.

1. Mumford's curves. Let $F$ be a nonsingular cubic surface in $\mathbf{P}^{3}, H$ the hyperplane divisor of $F, E$ any one of the 27 lines on $F$. Mumford's curves, $\gamma$, are the nonsingular members of the linear system $|4 H+2 E|$.

Wahl [7, §3.6] has proven the following:

Proposition 1.1. Any of Mumford's curves is defined by a homogeneous ideal $I=\left(F, S_{1}, S_{2}, S_{3}\right)$, where the $S_{i}$ are nonsingular sextic forms.

Actually the proof of the proposition shows that any member of $|4 H+2 E|$ is given by an ideal $I=\left(F, S_{1}, S_{2}, S_{3}\right)$ where the $S_{i}$ are sextic forms but not necessarily nonsingular. We will give the $S_{i}$ quite explicitly.

Let $V\left(f_{1}, \ldots, f_{n}\right)$ be the variety in $\mathbf{P}^{3}$ defined by the homogeneous polynomials $f_{1}, \ldots, f_{n}$.

Assume $E=V(x, y)$, changing co-ordinates if necessary. Then $F=x A+y B, A$ and $B$ quadratic forms. Since $F$ is nonsingular, $A$ and $B$ have no common component modulo the ideal $(x, y)$. In fact, if $L \mid A$ and $L \mid B(\bmod (x, y))$, the Jacobian criterion shows that $V(x, y, L)$ are singular points of $F$.

Proposition 1.2. $D \in|4 H+2 E|$ if and only if $D$ is defined by an ideal of the form $I(\eta, \xi)=\left(F, x^{2} \eta+B^{2} \xi, x y \eta-A B \xi, y^{2} \eta+A^{2} \xi\right), \eta$ a quartic form, $\xi a$ quadratic form.

Proof. We will show that ideals of the form $I(\eta, \xi)$ define elements of $14 H+$ $2 E \mid$, then show, by comparing dimensions, that divisors defined by such ideals are all of $|4 H+2 E|$.

For $\eta \notin(F)$, it is clear that $I(\eta, 0)$ defines an element of $|4 H+2 E|$.

If $\xi \neq 0$, let $g_{1}=x, g_{2}=y, g_{3}=A, g_{4}=B$. Let $W_{i}=\mathbf{P}^{3} \sim V\left(g_{i}\right)$, and $U_{i}=W_{i}$ $\cap F$. Let $D$ be given by $I(\eta, \xi)$.

On $U_{1}$, the Cartier divisor $D$ is defined by $f_{1}=S_{1} / x^{6}$; on $U_{2}$ by $f_{2}=S_{3} / y^{6}$; on $U_{3}$ by $f_{3}=S_{3} / A^{3}$; on $U_{4}$ by $f_{4}=S_{1} / B^{3}$.

We claim that $D$ is linearly equivalent to $D^{\prime}$ defined by $I(q, 0), q \notin(F)$. In fact, $D^{\prime}$ is defined on $U_{1}$ by $f_{1}^{\prime}=S_{1}^{\prime} / x^{6}=x^{2} q / x^{6}$; etc. It is easy to check that $f_{i} / f_{i}^{\prime}=S_{2} / S_{2}^{\prime}$, for all $i$. For example, on $U_{1}$, the quotient

$$
\begin{aligned}
S_{2} / S_{2}^{\prime} & =(x y \eta-A B \xi) / x y q=\left(x^{2} y \eta-x A B \xi\right) / x^{2} y q \\
& =\left(x^{2} y \eta+y B^{2} \xi\right) / x^{2} y q=\left(S_{1} / x^{6}\right) \cdot\left(x^{6} / S_{1}^{\prime}\right) .
\end{aligned}
$$

Thus, for all $i, f_{i} / f_{i}^{\prime}=f_{1} / f_{1}^{\prime}$ in $K$, and $D \equiv D^{\prime}$. Hence $D \in|4 H+2 E|$.

Now we have a map $\operatorname{Proj}\left(P_{4} \oplus P_{2}\right) \stackrel{\phi}{\rightarrow}|4 H+2 E|$ given by $\phi([\eta, \xi])=($ the divisor defined by $I(\eta, \xi))$. $\operatorname{Dim}|4 H+2 E|=37, \operatorname{Dim}\left(P_{4} \oplus P_{2}\right)=35+10=45$; 
thus $\operatorname{Dim}\left(\operatorname{Proj}\left(P_{4} \oplus P_{2}\right)\right)=44$. It is not hard to see that the kernel of $\phi$ is 7-dimensional, spanned by the 4-dimensional family $(\eta, 0), \eta \in(F)$, and the 3-dimensional family $(0, \xi)-\left(a B^{2}-b A B+c A^{2}, 0\right)$, where $\xi=a x^{2}+b x y+c y^{2}$. Thus $\operatorname{Dim}(\operatorname{im} \phi)=44-7=37$, so $\phi$ is onto.

Proposition 1.3. The relation module of $I(\eta, \xi)$ is generated by $r_{1}=$ $(-B \xi, y,-x, 0), r_{2}=(A \xi, 0, y,-x), r_{3}=(-x \eta, A, B, 0), r_{4}=(-y \eta, 0, A, B)$.

Proof. We claim the following sequence is exact:

$$
0 \rightarrow P(-9) \stackrel{\phi_{3}}{\rightarrow} P(-7)^{2} \oplus P(-8)^{2} \stackrel{\phi_{2}}{\rightarrow} P(-3) \oplus P(-6)^{3} \stackrel{\phi_{1}}{\rightarrow} I \rightarrow 0,
$$

where $\phi_{1}=\left(F, S_{1}, S_{2}, S_{3}\right)$,

$$
\phi_{2}=\left[\begin{array}{cccc}
-B \xi & A \xi & -x \eta & -y \eta \\
y & 0 & A & 0 \\
-x & y & B & A \\
0 & -x & 0 & B
\end{array}\right],
$$

$\phi_{3}=(A, B, y,-x)$. The exactness follows easily from [1, Corollary 2]. Then we observe that the relation module is generated by the columns of $\phi_{2}$.

Taking associated sheaves in the exact sequence above we obtain:

PROPOSITION 1.4. $\theta_{\gamma}$ has a resolution

$$
0 \rightarrow \mathcal{O}(-9) \rightarrow \mathcal{O}(-7)^{2} \oplus \mathcal{O}(-8)^{2} \rightarrow \mathcal{O}(-3) \oplus \vartheta(-6)^{3} \rightarrow \mathcal{\vartheta} \rightarrow \mathcal{\theta}_{\gamma} \rightarrow 0
$$

as an $\theta=\Theta_{\mathbf{P}^{3}-\text { module. }}$

2. Deformations of cones. Consider the Hilbert scheme $\mathbf{H}$ parametrizing subvarieties of $\mathbf{P}^{n}$ having the same Hilbert polynomial as a given subvariety $X$. In this section we will see that $\mathbf{H}$ is locally the same as the homogeneous deformations of the cones over $X$ defined by certain homogeneous ideals (Theorem 1).

As in [4], we let $C$ be the category of Artinian local $K$-algebras with residue field $k$ and $\hat{C}$ be the category of complete Noetherian local $k$-algebras $A$ for which $A / \underline{m}^{n}$ is in $C$ for all $n$. A functor on $C$ is prorepresentable if it is represented by an object of $\hat{C}$.

Let $X$ be a subscheme of $\mathbf{P}^{n}$. Let $I=\left(f_{1}(\underline{x}), \ldots, f_{m}(\underline{x})\right), f_{i}(x)$ homogeneous of degree $n_{i}$, be an ideal defining $X$. Define $V(I) \subset A^{n+1}$ to be the cone over $X$ defined by $I$. Note that $V(I)$ is different for different $I$ defining $X$.

Definition 1. Let $T \in C$ be given, its maximal ideal $M_{T}$. A deformation of the cone $V(I)$ over Spec $T$ is a closed subscheme $V$ of $A^{n+1} \times_{\text {Spec } k}$ Spec $T$, that is flat over Spec $T$, and is defined by $m$ equations $F_{i} \in T[x]$, homogeneous of degree $n_{i}$ in $\underline{x}$, such that $F_{i}=f_{i}(\underline{x}) \bmod \left(M_{T} T[\underline{x}]\right)$.

$V$ is flat over Spec $T$ if and only if it satisfies the lifting of relations condition: every relation $\left(r_{1}, \ldots, r_{m}\right)$ on $\left(f_{1}, \ldots, f_{m}\right)$, i.e. $\sum_{i=1}^{m} r_{i} f_{i}=0$, lifts to a relation $\left(R_{1}, \ldots, R_{m}\right)$ on $\left(F_{1}, \ldots, F_{m}\right)$, i.e. $\sum R_{i} F_{i}=0$ and $R_{i}=r_{i}(x) \bmod \left(M_{T} T[\underline{x}]\right)$.

Let $F(T)$ be the set of deformations of the cone $V(I)$ over Spec $T, T \in C$. Suppose we are given a map in $C, \phi: S \rightarrow T$, and $W \in F(S) . W$ is defined by $F_{i} \in S[\underline{x}]$. Now $\phi$ induces a natural map $\phi: S[\underline{x]} \rightarrow T[\underline{x}]$. Let $F(\phi)(W) \in F(T)$ be 
given by $F_{i}^{\prime}=\phi\left(F_{i}\right)$. (Flatness is easy to check using the lifting of relations $R_{j}^{\prime}=\phi\left(R_{j}\right)$.) Thus $F(\cdot)$ is a covariant functor on $C$.

Proposition 2.1. $F(\cdot)$ is prorepresentable.

Proof. We will use Theorem $2.11[4] . F(k)$ is a one point set, namely $\{V(I)\}$. Given $A^{\prime} \rightarrow A$ and $A^{\prime \prime} \rightarrow A$ in $C$, it is easy to see that $\left(A^{\prime} \times_{A} A^{\prime \prime}\right)[x] \rightarrow A^{\prime}[\underline{x}]$ $\times_{A[x]} A^{\prime \prime}[x]$ is an isomorphism. It follows that $F\left(A^{\prime} \times_{A} A^{\prime \prime}\right) \rightarrow F\left(A^{\prime}\right) \times_{F(A)} F\left(A^{\prime \prime}\right)$ is an isomorphism. This establishes $\left(H_{1}\right),\left(H_{2}\right)$, and $\left(H_{4}\right)$. The tangent space $t_{F}=F(k[\varepsilon])=\operatorname{Hom}_{P}(I, P / I)_{0}$ is finite dimensional, so $\left(H_{3}\right)$ holds. Then $F(\cdot)$ is prorepresentable.

Thus $F(\cdot)=h_{A}(\cdot)$, for some $A \in \hat{C}$.

Recall that $t_{F}$ is the Zariski tangent space to $\operatorname{Spec} A$, the space of infinitesimal deformations of $V(I)$.

Consider the algebras $k[t] /\left(t^{n}\right), n=2,3, \ldots$ Given an element $V$ of $F\left(k[t] /\left(t^{n}\right)\right)$ can it be extended to an element $V^{\prime}$ of $F\left(k[t] / t^{n+1}\right)$ ? That is, does there exist a $V^{\prime}$ such that $F(\phi)\left(V^{\prime}\right)=V$, where $\phi$ is the natural map $k[t] /\left(t^{n+1}\right) \rightarrow$ $k[t] /\left(t^{n}\right)$. Thus we have equations $f_{i}(\underline{x} ; t) \bmod \left(t^{n}\right)$ for $V$, so that for any relation $r_{i}(\underline{x} ; t), \sum f_{i}(\underline{x} ; t) r_{i}(\underline{x} ; t)=0 \bmod \left(t^{n}\right)$. To get $V^{\prime}$, we need $\Delta f_{i}$ and $\Delta r_{i}$, homogeneous of the same degree in $\underline{x}$ as $f_{i}$ and $r_{i}$, so that

$$
\sum\left(f_{i}+t^{n} \Delta f_{i}\right)\left(r_{i}+t^{n} \Delta r_{i}\right)=0 \bmod \left(t^{n+1}\right) .
$$

We have a presentation of $I=\left(f_{1}, \ldots, f_{m}\right)$ :

$$
0 \rightarrow R \stackrel{\beta}{\rightarrow} \bigoplus P\left(-n_{i}\right) \stackrel{\alpha}{\rightarrow} I \rightarrow 0 \quad\left(n_{i}=\operatorname{deg} f_{i}\right),
$$

where $\alpha$ and $\beta$ are homomorphisms of degree 0 . Then $r_{i}(x) \mapsto t^{n}$-coefficient of $\sum f_{i}(\underline{x} ; t) r_{i}(\underline{x} ; t)$ defines an obstruction map $R \rightarrow P / I . V$ extends to a $V^{\prime}$ if and only if the obstruction map lifts to a homomorphism $\delta: \bigoplus P\left(-n_{i}\right) \rightarrow P / I$. Both the obstruction map and its lifting $\delta$ (if it exists) are homomorphisms of degree 0 . The obstruction map vanishes on $R_{0}$, the submodule of $R$ of trivial relations (i.e., those of the form $\alpha(a) b-\alpha(b) a)$.

Define $T^{2}(I)$ by

$$
\operatorname{Hom}\left(\oplus P\left(-n_{i}\right), P / I\right)_{0} \rightarrow \operatorname{Hom}\left(R / R_{0}, P / I\right)_{0} \rightarrow T^{2}(I) \rightarrow 0 .
$$

Then $T^{2}(I)$ contains the obstructions to lifting deformations from $k[t] /\left(t^{n}\right)$ to $k[t] /\left(t^{n+1}\right)$, for any $n$.

We now restrict our attention to certain ideals defining $X$. Fix an $I$ maximal among all (homogeneous) ideals defining $X$ in $\mathbf{P}^{n} . V(I)$ for any such $I$ is called the minimal cone of $X$ and is characterized by $(x) \notin \operatorname{Ass}(P / I)$. Since $I$ is homogeneous, $I=\bigoplus_{j=0}^{\infty} I_{j}, I_{j}=$ forms of degree $j$ in $I$. Define $I_{(n)}=\bigoplus_{j=n}^{\infty} I_{j}$. The $I_{(n)}$ are also ideals defining $X$, while their cones contain $V(I)$.

Define $T_{n}^{1}=\operatorname{Hom}_{p}\left(I_{(n)}, P / I\right)_{0}$. If $n \leqslant m$ the natural inclusion $I_{(m)} \rightarrow I_{(n)}$ induces a $\operatorname{map} T_{n}^{1} \rightarrow T_{m}^{1}$.

Proposition 2. If ( $\underline{x}) \notin \operatorname{Ass} P / I$, then $(\underline{x}) \notin \operatorname{Ass}(\operatorname{Hom}(M, P / I))$ for any graded $P$-module $M$. 
Proof. Suppose $(x) \in \operatorname{Ass}(\operatorname{Hom}(M, P / I))$ for some $P$-module $M$. Then there is a $\phi \in \operatorname{Hom}(M, P / I)$ such that $(x)=\operatorname{ann}(\phi)$. In particular $\phi \neq 0$, so that $\phi(m) \neq 0$ for some $m \in M$. Then $(\underline{x} \subseteq \subseteq \operatorname{ann}(\phi(m))$ and, being maximal, (x) must equal $\operatorname{ann}(\phi(m))$, so that $(\underline{x}) \in \operatorname{Ass}(P / I)$.

Corollary 2.1. For $n \leqslant m, T_{n}^{1} \rightarrow T_{m}^{1}$ is injective.

Proof. Suppose not. Then there is a $\phi \in T_{n}^{1}$ that goes to the zero map in $T_{m}^{1}$. Thus $\phi(b)=0$ if $\operatorname{deg} b \geqslant m$. Therefore $(\underline{x})^{m} \phi=0$ in $T_{n}^{1}$ so that $(\underline{x}) \in$ $\operatorname{Ass}\left(\operatorname{Hom}\left(I_{(n)}, P / I\right)\right)$ which contradicts the proposition.

Corollary 2.2. If $(\underline{x}) \notin \operatorname{Ass}(P / I)$, the usual map

$$
\alpha: \operatorname{Hom}(M, P / I)_{0} \rightarrow H^{0}\left((\operatorname{Hom}(M, P / I))^{\sim}\right)
$$

is injective (where $\tilde{M}$ means the sheaf associated to $M$ ).

Proof. In [6, 67] Serre shows that the map $M \rightarrow \sum H^{0}(\tilde{M}(n))$ is injective if $(x) \notin \operatorname{Ass}(M)$.

Thus, in particular, the following map is injective:

$$
T_{n}^{1} \rightarrow H^{0}\left((\operatorname{Hom}(I, P / I))^{\sim}\right)=H^{0}\left(N_{X}\right) \text { for any } n \text {. }
$$

We will use these results to compare the deformation theory of the cone to that of $X$ in $\mathbf{P}^{n}$.

Let $S_{n}$ be the representative of the functor of deformations of the cone $V\left(I_{(n)}\right)$. Spec $S_{n}$ is the parameter space for deformations of this cone.

Let $S$ be the local ring of the Hilbert scheme $\mathbf{H}$ at the point corresponding to $X$. Spec $S$ parametrizes deformations of $X$ over schemes $\operatorname{Spec} T, T \in C$.

Let $t$ (resp. $t_{n}$ ) denote the Zariski tangent space and $O$ (resp. $O_{n}$ ) the obstruction space (see, e.g., [5, p. 153]) of $\operatorname{Spec} S$ (resp. Spec $S_{n}$ ).

$$
\begin{array}{cc}
t=H^{0}\left(N_{X}\right), & t_{n}=T_{n}^{1}, \\
O \hookrightarrow H^{1}\left(N_{X}\right), & O_{n} \hookrightarrow T_{n}^{2} .
\end{array}
$$

Any deformation of the cone $V\left(I_{(n)}\right)$ over Spec $T, T \in C$, induces a deformation of $X$ over Spec $T$. This defines a map $\phi:$ Spec $S_{n} \rightarrow \operatorname{Spec} S$.

THEOREM 1. Let $X$ be a local complete intersection. It is well known that there is an $N$ such that for $n \geqslant N,(P / I)_{n} \stackrel{\sim}{\rightarrow} H^{0}\left(\vartheta_{X}(n)\right)$. For $n \geqslant N, \phi:$ Spec $S_{n} \rightarrow \operatorname{Spec} S$ is an isomorphism.

Proof. For any $n$ we have a presentation

$$
0 \rightarrow R \rightarrow \bigoplus P\left(-n_{i}\right) \rightarrow I_{(n)} \rightarrow 0, \quad n_{i} \geqslant n .
$$

Apply $\operatorname{Hom}(\cdot, P / I)$ to get

$$
0 \rightarrow \operatorname{Hom}\left(I_{(n)}, P / I\right) \rightarrow \bigoplus P / I\left(n_{i}\right) \rightarrow \operatorname{Hom}(R, P / I) \text { exact. }
$$

Take associated sheaves; then

$$
0 \rightarrow N_{X} \rightarrow \bigoplus \vartheta_{X}\left(n_{i}\right) \rightarrow \operatorname{Hom}(R, P / I)^{\sim} \text { is exact. }
$$


Call the cokernel $Q$. Thus

$$
0 \rightarrow N_{X} \rightarrow \bigoplus \vartheta_{X}\left(n_{i}\right) \rightarrow Q \rightarrow 0
$$

is an exact sequence of $\theta_{X}$-modules.

Consider the exact sequence

$$
0 \rightarrow \operatorname{Hom}\left(I_{(n)}, P / I\right) \rightarrow \bigoplus P / I\left(n_{i}\right) \rightarrow \operatorname{Hom}\left(R / R_{0}, P / I\right) \rightarrow S \rightarrow 0
$$

whose degree zero part is

$$
0 \rightarrow T_{n}^{1} \rightarrow\left(\bigoplus P / I\left(n_{i}\right)\right)_{0} \rightarrow \operatorname{Hom}\left(R / R_{0}, P / I\right)_{0} \rightarrow T_{n}^{2} \rightarrow 0 .
$$

Passing to associated sheaves

$$
0 \rightarrow N_{X} \rightarrow \bigoplus \mathcal{O}_{X}\left(n_{i}\right) \rightarrow\left(\operatorname{Hom}\left(R / R_{0}, P / I\right)_{0}\right)^{\sim} \rightarrow \tilde{S} \rightarrow 0 \text { is exact. }
$$

Since $X$ is a local complete intersection, and locally $\tilde{S}$ is $T_{2}$, which is 0 [5, p. 150], it follows that $\tilde{S}$ is the zero sheaf. Therefore

$$
0 \rightarrow N_{X} \rightarrow \bigoplus \vartheta_{X}\left(n_{i}\right) \rightarrow\left(\operatorname{Hom}\left(R / R_{0}, P / I\right)\right)^{\sim} \rightarrow 0 \text { is exact. }
$$

From (1) and (3) we have $Q=\left(\operatorname{Hom}\left(R / R_{0}, P / I\right)\right)^{\sim}$.

Taking cohomology in (1) for the top row and taking (2) as the bottom row we have

$$
\begin{aligned}
& 0 \rightarrow H^{0}\left(N_{X}\right) \rightarrow \bigoplus H^{0}\left(\mathcal{O}_{X}\left(n_{i}\right)\right) \rightarrow \quad H^{0}(Q) \quad \rightarrow H^{1}\left(N_{X}\right) \\
& \phi_{1} \uparrow \quad \phi_{2} \uparrow \quad \phi_{3} \uparrow \quad \phi_{4} \uparrow \\
& 0 \rightarrow T_{n}^{1} \rightarrow \bigoplus(P / I)_{n_{i}} \rightarrow \operatorname{Hom}\left(R / R_{0}, P / I\right)_{0} \rightarrow \quad T_{n}^{2} \rightarrow 0
\end{aligned}
$$

The maps $\phi_{1}, \phi_{2}$, and $\phi_{3}$ are injective by Corollary 2.2. For $n \geqslant N, \phi_{2}$ is an isomorphism. It follows easily that $\phi_{1}$ is surjective (hence an isomorphism) and that $\phi_{4}$ is injective.

Thus we have $t_{n} \stackrel{\sim}{\rightarrow} t$, and

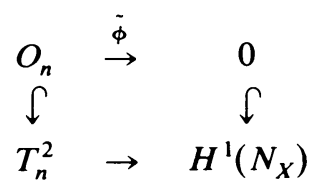

so that $\tilde{\phi}$ is injective. By the proposition on p. 153 of [5] $\phi$ is an isomorphism. Note that although the proposition is stated for local complex spaces the proof works over any field.)

This theorem means that the study of the local structure of the Hilbert scheme $\mathbf{H}$ is equivalent to the study of deformations of the cone $V\left(I_{(n)}\right)$ for large enough $n$.

3. The unobstructed deformations of Mumford's curves. For one of Mumford's curves it is not hard to see that $N=6$ (use Riemann-Roch). If $\gamma$ is defined by $I(\eta, \xi)$ on $F=x A+y B$, then deformations of $V(I)$ represent the case $N=3$. From diagram (4) we can compute that the cokernel of $\phi_{1}$ is one-dimensional. Since $\operatorname{dim} H^{0}\left(N_{\gamma}\right)=57$, the deformations of the cone $V(I)$ represent a 56-dimensional subfamily of the deformations of $\gamma$ in $\mathbf{P}^{3}$.

The ideal $I$ can be written in a determinantal form. 
Let

$$
M=\left[\begin{array}{cccc}
x & y & B \xi & -A \xi \\
-B & A & x \eta & y \eta
\end{array}\right]
$$

Define $M_{i j}=$ determinant of the $2 \times 2$ matrix formed by columns $i$ and $j$. For convenience let $M=\left(a_{i j}\right)$. The following two propositions are easily established.

Proposition 3.1. (a) $I$ is generated by the $M_{i j}$.

$$
\begin{array}{ll}
r_{1}=\left(-a_{13}, a_{12},-a_{11}, 0\right), & r_{2}=\left(a_{14}, 0, a_{12},-a_{11}\right), \\
r_{3}=\left(-a_{23}, a_{22},-a_{21}, 0\right), & r_{4}=\left(-a_{24}, 0, a_{22},-a_{21}\right) .
\end{array}
$$

Proposition 3.2. Let $N=\left(a_{i j}\right)$ be a $2 \times 4$ matrix over any commutative ring. Then

$$
\begin{aligned}
& -a_{13} N_{12}+a_{12} N_{13}-a_{11} N_{23}=0, \quad a_{14} N_{12}+a_{12} N_{23}-a_{11} N_{24}=0, \\
& -a_{23} N_{12}+a_{22} N_{13}-a_{21} N_{23}=0, \quad-a_{24} N_{12}+a_{22} N_{23}-a_{21} N_{24}=0 .
\end{aligned}
$$

Perturb $A, B, x, y, \xi$, and $\eta$ in $M$ as follows. Let

$$
M(t)=\left(\begin{array}{cccc}
x+t c & y+t d & (B+t b)(\xi+t e) & -(A+t a)(\xi+t e) \\
-B-t b & A+t a & (x+t c)(\eta+t f) & (y+t d)(\eta+t f)
\end{array}\right),
$$

where $a, b, c, d, e, f$ are homogeneous of the same degree as $A, B, x, y, \xi, \eta$, respectively. Let $I(t)$ be the ideal generated by the $M(t)_{i j} . I(0)=I$, and Proposition 2 gives the lifting of the relations $r_{1}, r_{2}, r_{3}, r_{4}$. This gives a flat family of the cone $V(I)$ over $k(t)$, and hence an unobstructed deformation of $\gamma$ in $\mathbf{P}^{3}$.

Any cubic form is in the ideal $(x, y, A, B)$. This, together with Proposition 1.2, guarantees that any element of $|4 H+2 E|$ or any cubic is $M(1)$ for some choice of $a, \ldots, f$.

Thus the $M(t)$ account for all deformations of $V(I)$ and of $\gamma$ in $\mathbf{P}^{3}$ over the parameter space $\operatorname{Spec} k(t)$.

4. Obstructed deformations. The dimension of Hilb at $\gamma$ is 56 , while the dimension of its tangent space, $H^{0}\left(N_{\gamma}\right)$, is $57 . H^{0}\left(N_{\gamma}\right)$ represents infinitesimal deformations of $\gamma$. There must be infinitesimal deformations that are obstructed.

$N_{X, Y}$ is the normal sheaf of $X$ in $Y$. As in [3] define $N_{F}=N_{\gamma, F}, N_{P}=N_{F,\left.P^{3}\right|_{\gamma}} \simeq$ $\vartheta_{\gamma}(3 h), h=H \cdot \gamma$. Let $N_{\gamma}=N_{\gamma, P^{3}}$. The sequence $0 \rightarrow N_{F} \rightarrow N_{\gamma} \rightarrow N_{P} \rightarrow 0$ is exact. Thus $0 \rightarrow N_{F} \rightarrow N_{\gamma} \rightarrow \mathcal{O}_{\gamma}(3 h) \rightarrow 0$ is exact. Taking cohomology; we get the exact sequence

$0 \rightarrow H^{0}\left(N_{F}\right) \rightarrow H^{0}\left(N_{\gamma}\right) \rightarrow H^{0}\left(\vartheta_{\gamma}(3 h)\right) \rightarrow H^{1}\left(N_{F}\right) \rightarrow H^{1}\left(N_{\gamma}\right) \rightarrow H^{1}\left(\vartheta_{\gamma}(3 h)\right) \rightarrow 0$.

$N_{F} \cong \theta_{\gamma}(\gamma \cdot \gamma) \cong \theta_{\gamma}\left(K_{\gamma}+h\right)$, hence $H^{1}\left(N_{F}\right)=0$. Thus $0 \rightarrow H^{0}\left(N_{F}\right) \rightarrow H^{0}\left(N_{\gamma}\right) \rightarrow$ $H^{0}\left(\vartheta_{\gamma}(3 h)\right) \rightarrow 0$ and $0 \rightarrow H^{1}\left(N_{\gamma}\right) \rightarrow H^{1}\left(\vartheta_{\gamma}(3 h)\right) \rightarrow 0$ are exact.

The sections of $N_{\gamma}$ coming from sections of $N_{F}$ correspond to infinitesimal deformations of $\gamma$ in $F$, i.e., members of $|4 H+2 E|$. These are unobstructed. Thus the obstructed deformations of $\gamma$ must correspond to sections of $N_{\gamma}$ coming from $H^{0}\left(\vartheta_{\gamma}(3 h)\right)$. Those sections of $\vartheta_{\gamma}(3 h)$ that are given by one cubic polynomial on $\gamma$ 
(i.e., $\left.\operatorname{im}\left(H^{0}\left(\mathcal{\vartheta}_{\mathbf{P}^{3}}(3)\right) \rightarrow H^{0}\left(\mathcal{O}_{\gamma}(3 h)\right)\right)\right)$ are also unobstructed. Thus an obstructed deformation must correspond to an element of $H^{0}\left(\vartheta_{\gamma}(3 h)\right)$ not induced by an element of $H^{0}\left(\vartheta_{\mathbf{p}^{3}}(3)\right)$.

Since $\gamma$ is nonsingular, the ring $\sum_{n=0}^{\infty} H^{0}\left(\Theta_{\gamma}(n h)\right)$ is the integral closure of the projective co-ordinate ring of $\gamma, P / I$. Elements of $H^{0}\left(\vartheta_{\gamma}(n h)\right)$ may be thought of as elements of the quotient field $K$ of $P / I$, of degree $n$, and integral over $P / I$.

Let $u=-B \xi / x .(\xi \neq 0$ or else $\gamma$ is singular. $)$

$$
\operatorname{Deg} u=3 \text { in } K, \quad u^{2}=B^{2} \xi^{2} / x^{2}=-x^{2} \eta \xi / x^{2}=-\eta \xi
$$

so $u$ is integral over $P / I$, but not in $P / I$. Thus $u$ should lead to an obstructed deformation of $\gamma$. Note that

$$
u=-B \xi / x=A \xi / y=-y \eta / A=x \eta / B \text { in } K .
$$

LEMMA 4.1. $(P / I)_{n} \rightarrow H^{0}\left(\Theta_{\gamma}(n h)\right)$ is an isomorphism for $n \geqslant 6$, not for $n=$ $3,4,5$.

Proof. Using the Riemann-Roch theorem we have

$$
\begin{gathered}
\operatorname{dim} H^{0}\left(\mathcal{O}_{\gamma}(3 h)\right)=20, \quad \operatorname{dim} H^{0}\left(\mathcal{O}_{\gamma}(n h)\right)=14 n-23, \quad n>3, \\
\operatorname{Dim}(P / I)_{n}=\operatorname{dim} P_{n}-\operatorname{dim} I_{n}=\left(\begin{array}{c}
n+3 \\
3
\end{array}\right),
\end{gathered}
$$

and the ideal $I=\left(F, S_{1}, S_{2}, S_{3}\right), F$ cubic, $S_{i}$ sextic. Thus

$$
\operatorname{dim} I_{n}= \begin{cases}\left(\begin{array}{l}
n \\
3
\end{array}\right), & n=3,4,5, \\
14 n-23, & n \geqslant 6 .\end{cases}
$$

Thus the map $(P / I)_{n} \rightarrow H^{0}\left(\vartheta_{\gamma}(n h)\right)$, injective since $(\underline{x}) \notin \operatorname{Ass}(P / I)$, is surjective if $n \geqslant 6$, but not for $n=3,4,5$.

Proposition 4.2. The deformation theories of $\gamma$ in $\mathbf{P}^{3}$ and of $V\left(I_{(n)}\right)$ are the same for $n \geqslant 6$, different for $n<6$.

Proof. The lemma and Theorem 1 of $\$ 2$ give the results for $n \geqslant 6$. For $n \leqslant 3$, $I_{(n)}=\left(F, S_{1}, S_{2}, S_{3}\right)$. Take the resolution $0 \rightarrow R \rightarrow P(-3) \oplus P(-6)^{3} \rightarrow I_{(n)} \rightarrow 0$. The map

$$
\phi_{2}: P_{3} \oplus P_{6}^{3} \rightarrow H^{0}\left(\vartheta_{\gamma}(3 h)\right) \oplus H^{0}\left(\vartheta_{\gamma}(6 h)\right)^{3}
$$

is not surjective by the lemma. Thus the map $\phi_{1}: T_{n}^{1} \rightarrow H^{0}\left(N_{\gamma}\right)$ is not surjective. Thus the deformation theories are different.

The cases $n=4,5$ are similar.

Let $\underline{\sigma}=\left(x^{3}, x^{2} y, \ldots, w^{3}\right)$ be the 20-tuple of cubic monomials in $P$. Then $I_{(6)}=\left(F \underline{\sigma} ; S_{1}, S_{2}, S_{3}\right) . I_{(6)}$ has the resolution $0 \rightarrow R \rightarrow P(-6)^{23} \rightarrow I_{(6)} \rightarrow 0$.

$R$ is generated by two types of relations:

(i) relations among the cubics, i.e. $r=(\underline{r} ; 0,0,0), \underline{r} \cdot \underline{\sigma}=0$;

(ii) relations induced by those on $I$. 
Let $B \xi=\underline{a} \cdot \underline{\sigma}, A \xi=\underline{b} \cdot \underline{\sigma}, \eta=\underline{c} \cdot \underline{\sigma}$; then the latter are generated by

$$
\begin{array}{ll}
r_{1}=(-\underline{a} ; y,-x, 0), & r_{2}=(\underline{b} ; 0, y,-x), \\
r_{3}=(-x \underline{c} ; A, B, 0), & r_{4}=(-y \underline{c} ; 0, A, B) .
\end{array}
$$

A deformation of $V(I)$ is given by $\Delta f=\left(G, T_{1}, T_{2}, T_{3}\right), G$ cubic, $T_{i}$ sextic. This yields a deformation of $I_{(6)}$ defined by $\left(G \underline{\sigma} ; T_{1}, T_{2}, T_{3}\right)$.

For any cubic $H, H u \in P$ (since $(x, y, A, B) u \in P$ and $H \in(x, y, A, B)$ ). By abuse of notation we consider $H u \in P / I$. Thus $u \underline{\sigma} \in(P / I)^{20}$. We call $u \underline{\sigma}$ any choice of coset representatives of $u \underline{\sigma}$ in $P^{20}$. any two choices for $u \underline{\sigma}$ differ by an element of $I^{20}$.

Write $\eta=\alpha x+\beta y+\gamma A+\delta B, \operatorname{deg} \alpha=\operatorname{deg} \beta=3, \operatorname{deg} \gamma=\operatorname{deg} \delta=2$.

Proposition 4.3. Let $T_{1}=(2 \beta x-2 \gamma B) \xi, T_{2}=(-\alpha x+\beta y+\gamma A-\delta B), T_{3}=$ $(2 \delta A-2 \alpha y) \xi$. For any choice of $u \underline{\sigma}, \Delta f=\left(u \underline{\sigma} ; T_{1}, T_{2}, T_{3}\right)$ defines an infinitesimal deformation of $V\left(I_{(6)}\right)$, hence of $\gamma$.

Proof. It is only necessary to check that $\Delta f \cdot r \in I$ for all $r \in R$. E.g.,

$$
\begin{aligned}
\Delta f \cdot r_{4} & =-y u \underline{\sigma} \cdot \underline{c}+A T_{2}+B T_{3}=-y u \eta+A T_{2}+B T_{3} \\
& =-y(-\alpha B \xi+B A \xi-\gamma y \eta+\delta x y)+A T_{2}+B T_{3} \bmod I,
\end{aligned}
$$

since $x u=B \xi \bmod I$, etc. It is then easy to see $\Delta f \cdot r_{4}=0 \bmod I$. The rest are similar.

Note that $T_{1}, T_{2}, T_{3}$ depend only on $\eta$ and $\xi$, not on the choice of $u$.

Any section of $H^{0}\left(\theta_{\gamma}(3 h)\right)$ is given by $G+m u, G \in P_{3}, m \in k$. Thus any infinitesimal deformation of $\gamma$ comes from a $\Delta f=\left((G+m u) \underline{\sigma}, T_{1}^{\prime}, T_{2}^{\prime}, T_{3}^{\prime}\right)$ where the $T_{i}^{\prime}$ are $T_{i}$ plus the corresponding sextic for $G$ (see $\S 3$ ).

5. The order of obstruction. The obstructions to extending infinitesimal deformations of $\gamma$ lie in $H^{1}\left(N_{\gamma}\right) \stackrel{\sim}{\rightarrow} H^{1}\left(\vartheta_{\gamma}(3 h)\right)$. It is convenient to observe that $H^{1}\left(\vartheta_{\gamma}(3 h)\right)$ can be computed via a Koszul complex.

Define $D(f)=\mathbf{P}^{3} \sim V(f), f \in P$. Then $D(x), D(y), D(A), D(B)$ is an affine cover of $\gamma$, so that the cohomology of $\gamma$ can be computed via the standard Čech resolution for this cover.

$L=\sum H^{0}\left(\Theta_{\gamma}(n)\right)$ is the integral closure of $P / I$. Let $M=L(3)$; thus the associated sheaf $\tilde{M}=\theta_{\gamma}(3 h)$.

The sequence $(x, y, A, B)$ is regular on $P$. Let $K=K .(x, y, A, B)$ be the graded Koszul complex

$$
\begin{aligned}
0 & \rightarrow P(-6) \rightarrow P(-5)^{2} \oplus P(-4)^{2} \rightarrow P(-2) \oplus P(-3)^{4} \oplus P(-4) \\
& \rightarrow P(-1)^{2} \oplus P(-2)^{2} \rightarrow P
\end{aligned}
$$

with the usual maps (see, e.g., [2, p. 245]). Let $C_{*}$ be the complex $\operatorname{Hom}(K, M)$, and $\left(C_{*}\right)_{0}$ the subcomplex of $C_{*}$ of degree zero.

Proposition 5.1. $H^{i}\left(\left(C_{*}\right)_{0}\right) \stackrel{\sim}{\rightarrow} H^{i}\left(\vartheta_{\gamma}(3 h)\right), i=0,1$. 
Proof. We use the following notation:

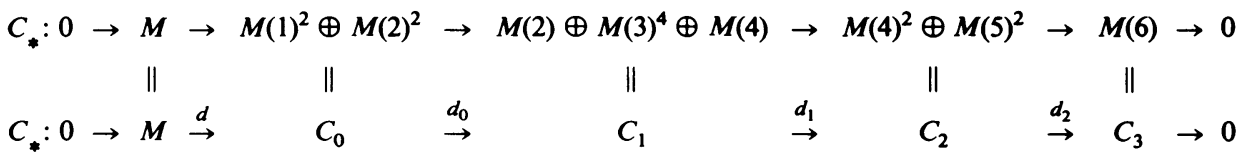

thus $H^{i}\left(\left(C_{*}\right)_{0}\right)=\left(\operatorname{Ker} d_{i}\right)_{0} /\left(\mathrm{im} d_{i-1}\right)_{0}$.

Taking associated sheaves we have the complex $\tilde{C}_{*}$ :

$$
0 \rightarrow \tilde{M} \stackrel{d^{\prime}}{\rightarrow} \tilde{C}_{0} \stackrel{d_{0}^{\prime}}{\rightarrow} \tilde{C}_{1} \stackrel{d_{1}^{\prime}}{\rightarrow} \tilde{C}_{2} \stackrel{d_{2}^{\prime}}{\rightarrow} \tilde{C}_{3} \rightarrow 0 .
$$

This complex is exact since $H^{i}\left(\tilde{C}_{*}\right)=\operatorname{Ext} t^{i}\left(\mathcal{O}_{\mathbf{p}^{3}}, \tilde{M}\right)$, which is 0 for $i>0$, and is $\tilde{M}$ for $i=0$.

Let $Q=\operatorname{im} d_{0}^{\prime}=\operatorname{ker} d_{1}^{\prime}$. Then $0 \rightarrow \tilde{M} \rightarrow \tilde{C}_{0} \rightarrow Q \rightarrow 0$ is exact. Taking cohomology,

$$
0 \rightarrow H^{0}(\tilde{M}) \rightarrow H^{0}\left(\tilde{C}_{0}\right) \rightarrow H^{0}(Q) \rightarrow H^{1}(\tilde{M}) \rightarrow 0 .
$$

The final zero is $H^{1}\left(\tilde{C}_{0}\right)$, zero since $H^{1}\left(\vartheta_{\gamma}(n h)\right)=0$ for $n>4$ ( $n h$ is nonspecial).

The usual map $f: M \rightarrow \Sigma H^{0}(\tilde{M}(n))$ is an isomorphism. Thus in the following diagram (where $\left.S=\left(\operatorname{ker} d_{1}\right)_{0} /\left(\operatorname{im} d_{0}\right)_{0}\right)$.

$$
\begin{aligned}
& 0 \rightarrow H^{0}(\tilde{M}) \quad \rightarrow \quad H^{0}\left(\tilde{C}_{0}\right) \quad \rightarrow \quad H^{0}(Q) \rightarrow H^{1}(\tilde{M}) \quad \rightarrow \quad 0 \\
& f \uparrow \quad f_{0} \uparrow \quad f_{1} \uparrow \quad f_{2} \uparrow \\
& 0 \rightarrow(M)_{0} \quad \rightarrow \quad\left(C_{0}\right)_{0} \quad \stackrel{d_{0}}{\rightarrow}\left(\operatorname{ker} d_{1}\right)_{0} \quad \rightarrow \quad S \quad S \quad \rightarrow \quad 0
\end{aligned}
$$

$f$ and $f_{0}$ are isomorphisms. We also have $0 \rightarrow Q \rightarrow \tilde{C}_{1} \rightarrow \tilde{C}_{2}$ where

$$
\begin{array}{cccccccc}
0 & \rightarrow & H^{0}(Q) & \rightarrow & H^{0}\left(\tilde{C}_{1}\right) & \rightarrow & H^{0}\left(\tilde{C}_{2}\right) \\
& & f_{1} \uparrow & & f_{3} \uparrow & & f_{4} \uparrow \\
0 & \rightarrow & \left(\operatorname{ker} d_{1}\right)_{0} & \rightarrow & \left(C_{1}\right)_{0} & \rightarrow & \left(C_{2}\right)_{0}
\end{array}
$$

where $f_{3}$ and $f_{4}$ are isomorphisms, hence $f_{1}$ is also an isomorphism. Then $S=$ $H^{1}\left(\left(C_{*}\right)_{0}\right) \stackrel{\sim}{\rightarrow} H^{1}\left(\theta_{\gamma}(3 h)\right)$.

The curve $\gamma$ is a local complete intersection, that is, there are $g_{1}, \ldots, g_{n} \in P$ so that on $D\left(g_{i}\right)=W_{i}$, the curve $\gamma$ is $V\left(f_{i}, s_{i}\right)$; with $f_{i}, s_{i} \in P_{\left(g_{i}\right)}$.

To find the element of $H^{1}\left(N_{\gamma}\right)$ that is the obstruction to lifting the deformation given by $\Delta f$, we change our point of view slightly. The curve $\gamma$ is defined by the ideal $\left(f_{i}, s_{i}\right)$ on $W_{i}=D\left(g_{i}\right)$. To extend the deformation of $\gamma$ it is enough to find extensions of the deformation over each $W_{i}$ by functions of degree zero such that the resulting perturbed ideals agree on the overlaps.

Let $W_{i j}=W_{i} \cap W_{j}$. We have $\left(u_{i}, v_{i}\right)$ such that

$$
\left(f_{i}+t u_{i}, s_{i}+t v_{i}\right)=\left(f_{j}+t u_{j}, s_{i}+t v_{j}\right) \bmod \left(t^{2}\right) \text { over } W_{i j} .
$$

We seek $\left(u_{i}^{\prime}, v_{i}^{\prime}\right)$ such that

$$
\left(f_{i}+t u_{i}+t^{2} u_{i}^{\prime}, s_{i}+t v_{i}+t^{2} v_{i}^{\prime}\right)=\left(f_{j}+t u_{j}+t^{2} u_{j}^{\prime}, s_{j}+t v_{j}+t^{2} v_{j}^{\prime}\right) \bmod \left(t^{3}\right)
$$


on $W_{i j}$. Thus we want $\left(u_{i}^{\prime}, v_{i}^{\prime}\right)$ such that there are $a, b, c$, etc. such that

$$
\begin{aligned}
f_{j}+t u_{j}+t^{2} u_{j}^{\prime}= & \left(a+t a^{\prime}+t^{2} a^{\prime \prime}\right)\left(f_{i}+t u_{i}+t^{2} u_{i}^{\prime}\right) \\
& +\left(b+t b^{\prime}+t^{2} b^{\prime \prime}\right)\left(s_{i}+t v_{i}+t^{2} v_{i}^{\prime}\right) \bmod \left(t^{3}\right) \\
= & a f_{i}+b s_{i}+t\left(a u_{i}+b v_{i}+a^{\prime} f_{i}+b^{\prime} s_{i}\right) \\
& +t^{2}\left(a u_{i}^{\prime}+b v_{i}^{\prime}+a^{\prime} u_{i}+b^{\prime} s_{i}+a^{\prime \prime} f_{i}+b^{\prime \prime} s_{i}\right) \bmod \left(t^{3}\right)
\end{aligned}
$$

and

$$
\begin{aligned}
s_{j}+t v_{j}+t^{2} v_{j}^{\prime}= & \left(c+t c^{\prime}+t^{2} c^{\prime \prime}\right)\left(f_{i}+t u_{i}+t^{2} u_{i}^{\prime}\right) \\
& +\left(d+t d^{\prime}+t^{2} d^{\prime \prime}\right)\left(s_{i}+t v_{i}+t^{2} v_{i}^{\prime}\right) \bmod \left(t^{3}\right)
\end{aligned}
$$

or

$$
\begin{aligned}
& u_{j}^{\prime}=a u_{i}^{\prime}+b v_{i}^{\prime}+a^{\prime} u_{i}+b^{\prime} v_{i}+a^{\prime \prime} f_{i}+b^{\prime \prime} s_{i} \\
& v_{j}^{\prime}=c u_{i}^{\prime}+d v_{i}^{\prime}+c^{\prime} u_{i}+d^{\prime} v_{i}+c^{\prime \prime} f_{i}+d^{\prime \prime} s_{i}
\end{aligned}
$$

Note that

$$
\begin{gathered}
u_{j}=a u_{i}+b v_{i}+a^{\prime} f_{i}+b^{\prime} s_{i}, \\
v_{j}=c u_{i}+d v_{i}+c^{\prime} f_{i}+d^{\prime} s_{i} . \\
u_{j}^{\prime}=a^{\prime} u_{i}+b^{\prime} v_{i}+a u_{i}^{\prime}+b v_{i}^{\prime} \\
v_{j}^{\prime}=c^{\prime} u_{i}+d^{\prime} v_{i}+c u_{i}^{\prime}+d v_{i}^{\prime}
\end{gathered}
$$

Now $\left(\begin{array}{ll}a & b \\ c & d\end{array}\right)=N_{j i}$, so that

$$
S=\left[\left(\begin{array}{c}
a^{\prime} u_{i}+b^{\prime} v_{i} \\
c^{\prime} u_{i}+d^{\prime} v_{i}
\end{array}\right)\right] \in H^{1}\left(N_{\gamma}\right)
$$

must vanish if we are to solve for

$$
\left(\begin{array}{c}
u_{i}^{\prime} \\
v_{i}^{\prime}
\end{array}\right) \quad\left(s_{j i}=\left(\begin{array}{c}
u_{j}^{\prime} \\
v_{j}^{\prime}
\end{array}\right)-N_{j i}\left(\begin{array}{c}
u_{i}^{\prime} \\
v_{i}^{\prime}
\end{array}\right)\right) .
$$

For Mumford's $\gamma$ we know the map $H^{1}\left(N_{\gamma}\right) \rightarrow H^{1}\left(\theta_{\gamma}(3 h)\right)$ given by

$$
\left[\left(\begin{array}{l}
u_{i j} \\
v_{i j}
\end{array}\right)\right] \mapsto\left[u_{i j}\right]
$$

where $u_{i j}$ is the restriction of $u_{i}$ to $W_{i j}$, is an isomorphism, so we only need to compute the $u_{i j}=a^{\prime} u_{i}+b^{\prime} v_{i} \bmod \left(f_{i}, s_{i}\right)$.

It is not hard to exhibit an open cover of $\mathbf{P}^{3}$, such that $\gamma$ is defined by two equations on each open set.

Proposition 5.2. On $D(x),\left(F / x^{3}, S_{1} / x^{6}\right)$ defines $\gamma$; on $D(y),\left(F / y^{3}, S_{3} / y^{6}\right)$; on $D(z A), \quad\left(F / z A, S_{3} /(z A)^{2}\right) ; \quad$ on $D(w A), \quad\left(F / w A, S_{3} /(w A)^{2}\right) ; \quad$ on $D(z B)$, $\left(F / z B, S_{1} /(z B)^{2}\right)$; on $D(w B),\left(F / w B, S_{1} /(w B)^{2}\right)$.

Proof. The relation $r_{1}$ gives $-B \xi F+y S_{1}-x S_{2}=0$. Thus, on $D(x)$,

$$
S_{2} / x^{6}=\left(B \xi / x^{4}\right)\left(F / x^{3}\right)+(y / x)\left(S_{1} / x^{6}\right)
$$

so $S_{2} / x^{6} \in\left(F / x^{3}, S_{1} / x^{6}\right)$. The rest follows similarly. 
Let $N_{21}$ be the transition matrix of the normal bundle on $D(x) \cap D(y) . N_{21}=$ $\left[\begin{array}{ll}a & b \\ c & d\end{array}\right]$, the solutions of

$$
F / y^{3}=a\left(F / x^{3}\right)+b\left(S_{1} / x^{6}\right), \quad S_{3} / y^{6}=c\left(F / x^{3}\right)+d\left(S_{1} / x^{6}\right) \bmod I .
$$

Thus

$$
N_{21}=\left[\begin{array}{cc}
x^{3} / y^{3} & 0 \\
2 x^{2} A \xi / y^{6} & x^{4} / y^{4}
\end{array}\right] .
$$

Consider $\Delta f=\left((G+m u) \underline{\sigma} ; T_{1}, T_{2}, T_{3}\right), m \neq 0$, as in $\S 4$. Under the map $\phi_{1}$ : $T_{6}^{1} \rightarrow H^{0}\left(N_{\gamma}\right), f$ goes to

$$
\left(\begin{array}{l}
u_{i} \\
v_{i}
\end{array}\right), \quad i=1, \ldots, 6
$$

where $u_{1}=-m B \xi / x^{4}+G / x^{3}, u_{2}=m A \xi / y^{4}+G / y^{3}$. Note that the map $H^{0}(N \gamma)$ $\rightarrow H^{0}\left(\Theta_{\gamma}(3 h)\right)$ is given by projection on the first factor.

LEMMA 5.3. $u_{12}=m A \xi^{2} / x y^{5}+G \xi / x y^{4}$, where $u_{12}$ is the restriction of $u_{1}$ to $W_{12}$.

Proof. From (2)

$$
\begin{aligned}
m A \xi / y^{4}+G / y^{3}= & \left(x^{3} / y^{3}\right)\left(-m B \xi / x^{4}+G / x^{3}\right) \\
& +a^{\prime}\left(F / x^{3}\right)+b^{\prime}\left(S_{1} / x^{6}\right) .
\end{aligned}
$$

So

$$
a^{\prime}\left(F / x^{3}\right)+b^{\prime}\left(S_{1} / x^{6}\right)=\left(x^{2} \xi / y^{4}\right)\left(F / x^{3}\right) .
$$

Take $a^{\prime}=x^{2} \xi / y^{4}, b^{\prime}=0$. From (4)

$$
u_{12}=a^{\prime} u_{1}+b^{\prime} v_{1}=m A \xi^{2} / x y^{5}+G \xi / x y^{4} .
$$

THEOREM 2. If $\xi \notin(x, y, A, B)$, then the cocycle $\left[u_{i j}\right] \neq 0$ in $H^{1}\left(\vartheta_{\gamma}(3 h)\right)$, hence the infinitesimal deformation $\Delta f$ does not extend to a deformation over $k[t] /\left(t^{3}\right)$.

Proof. Under the isomorphism $f_{2}: H^{1}\left(\left(C_{0}\right)_{0}\right) \rightarrow H^{1}\left(\vartheta_{\gamma}(3 h)\right),\left[u_{i j}\right]$ is the image of [ $\left.\phi_{i j}\right]$, where $\phi_{12}=m A \xi^{2} / y+G \xi=(u+G) \xi$. If $\left[\phi_{i j}\right]=0$, then $\phi_{12}=(u+G) \xi=$ $y \phi_{1}-x \phi_{2}$, where $\phi_{1}, \phi_{2} \in H^{0}\left(\theta_{\gamma}(4 h)\right)$. For $C \in P, C u \in P / I$ if and only if $C \in(x, y, A, B)$. Thus $y \phi_{1}-x \phi_{2} \in P / I$, while $u \xi \in P / I$. Thus $\left[\phi_{i j}\right] \neq 0$, hence $\left[u_{i j}\right] \neq 0$.

Thus if the curve $\gamma$ is defined by the ideal $\left(A x+B y, x^{2} \eta+B^{2} \xi, x y \eta-A B \xi\right.$, $\left.y^{2} \eta+A^{2} \xi\right)$, with $\xi \notin(x, y, A, B)$, then the infinitesimal deformation given by $\Delta f$ above does not extend to a deformation modulo $t^{2}$, i.e., it is obstructed to the second order.

\section{REFERENCES}

1. D. A. Buchsbaum and D. Eisenbud, What makes a complex exact?, J. Algebra 25 (1973), 259-268.

2. R. Hartshorne, Algebraic geometry, Graduate Texts in Math., vol. 52, Springer-Verlag, New York, 1977.

3. D. Mumford, Further pathologies in algebraic geometry, Amer. J. Math. 84 (1962), 642-648.

4. M. Schlessinger, Functors of Artin rings, Trans. Amer. Math. Soc. 130 (1968), 208-222.

5. __ On rigid singularities, Rice Univ. Studies 59 (1973), 147-162.

6. J.-P. Serre, Faiseaux algébriques cohérents, Ann. of Math. (2) 61 (1955), 197-278.

7. J. Wahl, Deformations of plane curves with nodes and cusps, Amer. J. Math. 96 (1974), 529-577.

Department of Mathematical Sciences, Northern Kentucky University, Highland Heights, KENTUCKY 41076 